\title{
Krankheitskosten sowie gesundheitsbezogene Lebensqualität (hrQoL) bei ambulant erworbener Pneumonie (CAP) - ein systematisches Review
}

\author{
Costs of Illness and Health-Related Quality of Life for Community-Acquired \\ Pneumonia - A Systematic Review
}

Autoren

Institute
C. Jacob', T. Mittendorf' , J.-M. Graf von der Schulenburg ${ }^{2}$

herescon gmbh, health economics research \& consulting, Hannover

Forschungsstelle für Gesundheitsökonomie, Gottfried Wilhelm Leibniz Universität Hannover, Hannover eingereicht 11.2.2011

akzeptiert nach Revision

7.3. 2011

Bibliografie

DOI http://dx.doi.org/

10.1055/s-0030-1256353

Online-Publikation: 21. 4. 2011

Pneumologie 2011; 65 :

498-502 ๔ Georg Thieme

Verlag KG Stuttgart · New York

ISSN 0934-8387

Korrespondenzadresse

Dipl..ök. Christian Jacob

herescon gmbh

health economics

research \& consulting

Königworther Str. 2

30167 Hannover

jacob@herescon.com

\section{Zusammenfassung \\ $\nabla$}

Zielsetzung: Für Deutschland liegen lediglich begrenzt Daten zu den Kosten und zur gesundheitsbezogenen Lebensqualität (hrQoL) der ambulant erworbenen Pneumonie (CAP) vor. Diese Arbeit untersucht, ob sich aus der internationalen Literatur umfassendere Erkenntnisse über die Krankheitskosten der CAP und die hrQoL bei CAP generieren lassen.

Methodik: Es wurde eine systematische Literaturrecherche in elektronischen Datenbanken durchgeführt. Die Selektion der Treffer erfolgte anhand der bibliografischen Angaben. Die eingeschlossene Literatur wurde getrennt nach Krankheitskosten- bzw. hrQoL-Analysen ausgewertet. Ergebnisse: Die Recherche ergab 172 Treffer, davon wurden 16 Publikationen zu Krankheitskosten der CAP und 5 Publikationen zu hrQoL bei CAP eingeschlossen. Lediglich zwei Publikationen sind aktuelleren Datums.

Schlussfolgerung: Es zeigt sich, dass nur wenige und veraltete Daten über die Krankheitskosten der CAP vorliegen und sich aufgrund des Studiendesigns die Studienergebnisse nicht auf Deutschland übertragen lassen. Zur Lebensqualität liegen keine verwertbaren Erkenntnisse vor, weswegen gefolgert werden kann, dass in diesem Bereich noch erheblicher Forschungsbedarf vorliegt.

\section{Hintergrund und Fragestellung}

Eine Pneumonie ist eine akute oder chronische Entzündung des Lungengewebes. Ambulant erworbene Pneumonien (CAP) treten gehäuft im höheren Lebensalter auf und gehören in Deutschland zu den zehn häufigsten Todesursachen bei Personen im Alter von 65 Jahren und älter. Die Bezeichnung „ambulant“ beschreibt dabei, dass die Infektion im privaten oder beruflichen Umfeld erworben wurde. Dahingegen steht eine nosoko-

\section{Abstract \\ $\nabla$}

Aim: There are only very limited data available on the costs and health-related quality of life (hrQoL) of patients with community-acquired pneumonia (CAP) in Germany. The aim of this review was to identify studies in the international literature regarding the costs and hrQoL of CAP.

Method: In March 2010, a systematic literature search was performed. The acquired literature was evaluated separately for costs of illness and/ or hrQoL analyses followed by a structured discussion of the findings.

Results: A total of 172 references was identified. Sixteen publications on the cost of illness for CAP and five publications on hrQoL met the selection criteria and were included in the present review. Conclusion: There are only very limited and, furthermore, outdated data available on the costs and quality of life impacts of CAP. Hence, further research is urgently needed to fill this lack of evidence. mial erworbene Pneumonie mit einem stationären Aufenthalt in einem Krankenhaus in Verbindung. Genaue Zahlen über die Inzidenz liegen nicht vor. Schätzungen gehen von ca. 400000 bis 600000 Neuerkrankungen pro Jahr in Deutschland aus [1]. Die Kosten, die im Zusammenhang mit Pneumonien für das deutsche Gesundheitssystem entstehen, lassen sich nur näherungsweise schätzen, da aus der Gesundheitsstatistik lediglich begrenzt Daten zu Behandlungskosten zur Verfügung stehen. Es wird vermutet, dass 
jährliche Kosten von über 500 Millionen Euro entstehen [2]. Auch liegen kaum Daten zur gesundheitsbezogenen Lebensqualität (hrQoL) bei CAP vor. Pneumonien sind in der klinischen Folge eine der Hauptkomplikationen von Pneumokokken-Infektionen, die insbesondere im höheren Erwachsenenalter einen entscheidenden Einfluss auf die Ergebnisse einer Kosten-EffektivitätsAnalyse zu im Markt verfügbaren Pneumokokken-Impfstoffen haben können.

Die nachfolgende Übersichtsarbeit geht der Frage nach, ob aus der internationalen Literatur umfassendere Erkenntnisse über die Krankheitskosten der CAP und die hrQoL bei CAP generiert werden können.

\section{Methodik \\ $\nabla$}

Zur Bewertung der Evidenz zu Krankheitskosten der CAP und hrQoL bei CAP wurde im März 2010 eine systematische Recherche in den elektronischen Literaturdatenbanken Ovid MEDLINE, Ovid MEDLINE In-Process, EMBASE und BIOSIS Previews durchgeführt. Dabei wurden in vier Suchstrategien unter Verwendung folgender Suchbegriffe Publikationen zu den Kosten der Pneumonie identifiziert: adolescent, adult, ambulant, ambulatory, community acquired pneumonia, cost, pneumonia, Germany, hospital, hospitalisation, hospitalization, inpatient, nursing home und outpatient. Diese wurden auf erwachsene Patienten, die englische oder deutsche Sprache, den Zeitraum von 1990 bis 2010 und Deutschland limitiert. Zur Identifikation von Publikationen zum Thema hrQoL bei CAP wurden die oben verwendeten Suchbegriffe um „quality of life“, „quality of living“ und „activities of daily life“ erweitert und die durchgeführte Recherche einmal mit und einmal ohne Limitationen durchgeführt. Die Selektion der Treffer erfolgte über die Auswertung der bibliografischen Angaben inklusive des Abstracts. Zusätzlich wurden Publikationen per Handrecherche identifiziert. Die Auswertung der eingeschlossenen Literaturtreffer erfolgte anhand der Volltexte getrennt nach Krankheitskostenanalysen und hrQoL.

\section{Ergebnisse}

\section{Systematische Literaturrecherche}

Insgesamt ergab die Literaturrecherche 157 Treffer plus 15 aus der Handrecherche. Ausgeschlossen wurden Abstracts, Kommentare usw. ( $n=13$ ), Publikationen, die nicht die Indikation CAP fokussierten $(\mathrm{n}=49)$, doppelte Treffer, Publikationen in anderen Sprachen als Deutsch oder Englisch $(n=34)$, Publikationen mit Fokus auf klinische Wirksamkeit oder Epidemiologie $(n=31)$, Versorgungsforschung $(\mathrm{n}=10)$ sowie die Kosten-Effektivität $(\mathrm{n}=12)$. Eine Übersicht über das Ergebnis der systematischen Recherche kann $\bullet$ Abb. 1 entnommen werden.

Für die detaillierte Literaturauswertung lagen 16 Publikationen zu Krankheitskosten der CAP vor, wovon acht aus der Handrecherche stammen. Zum Themenbereich gesundheitsbezogene Lebensqualität bei CAP wurden fünf Publikationen identifiziert.

\section{Krankheitskosten der CAP - Literaturauswertung}

- Tab. 1 gibt Auskunft über die wesentlichen Merkmale der eingeschlossenen Publikationen.

In $\bullet$ Tab. 2 finden sich Daten zur Studienperspektive, der Größe der Stichprobe sowie den ermittelten Kosten.

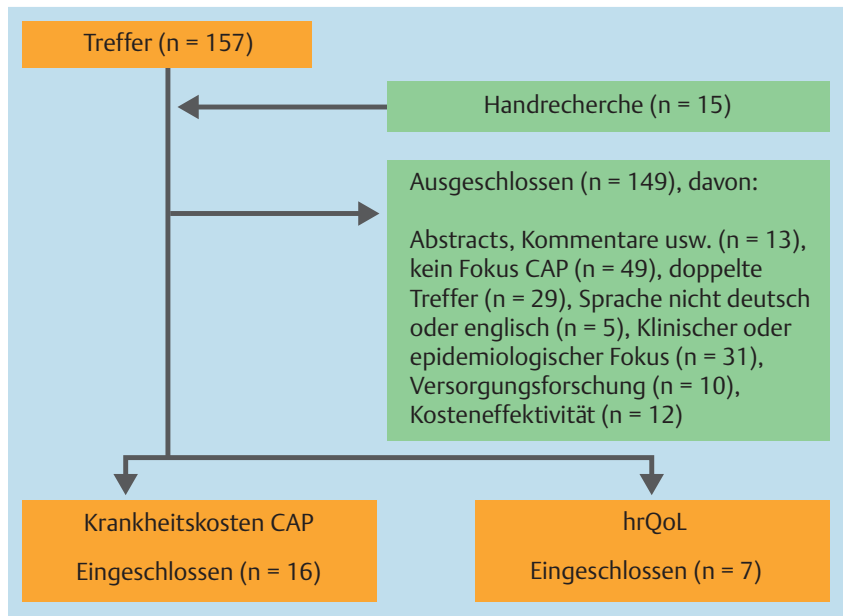

Abb. 1 Ergebnis der systematischen Literaturrecherche. CAP: community acquired pneumonia; hrQoL: health related Quality of Life.

Health related Quality of Life - Literaturauswertung In der Literaturrecherche wurden fünf Publikationen zu gesundheitsbezogener Lebensqualität bei CAP identifiziert. Zwei weitere Publikationen (Feagan [2001] [19] und Palmer et al. [2000] [20]) berichteten über die gleiche Studiengruppe wie die Autoren Marrie et al. (2000) [21]. Eine Übersicht über die eingeschlossenen Publikationen zeigt Tab. 3.

\section{Diskussion \\ $\nabla$}

In der Literaturrecherche fand sich lediglich eine aktuellere Publikation (jünger als fünf Jahre) zu den Krankheitskosten der CAP (siehe Tab. 1). Alle übrigen Publikationen $(n=15)$ sind aufgrund des Publikationsdatums als veraltet anzusehen, zumal der Zeitverzug zwischen Publikationsjahr und empirischem Datenjahr die Aktualität noch weiter beeinträchtigt. Allein zehn Kostenstudien wurden für die USA erstellt, während sich aus den Ländern AUS, D, E, NZ und UK nur je eine Kostenstudie fand. Die Ergebnisse der einzigen deutschen Publikation müssen allerdings angezweifelt werden.

Insgesamt 10 von 16 Publikationen konzentrierten sich auf die Versorgung von CAP im Krankenhaus. Das Studiendesign war entweder prospektiv als Beobachtungsstudie konzipiert oder es wurden retrospektiv Patientenakten analysiert. Drei Studien $[3,6,9]$ analysierten neben den stationären Kosten auch die Kosten der ambulanten Versorgung von Patienten mit CAP. Indirekte Kosten, insbesondere Arbeitsunfähigkeitstage, wurden in zwei Publikationen analysiert (Scott [17], NZ, und Birnbaum [5], USA). Ein großer Teil der Pflege wird in Deutschland von Pflegepersonen (entweder pflegende Angehörige, wie z.B. Ehepartner, andere Verwandte des Pflegebedürftigen oder Nachbarn bzw. Freunde) getragen. Häufig sind die Ehepartner Pflegepersonen, die ihrerseits altersbedingt ein hohes CAP-Risiko haben. Es fanden sich jedoch keine Studien über die indirekten Kosten der CAP, die aus einem CAP-bedingten Ausfall von Pflegepersonen resultieren könnten.

Keine Publikation adressierte die Kosten der Pflegebedürftigkeit in einer umfassenden Analyse. Es fand sich gleichwohl die Studie von Kruse et al. [12], die zusätzliche Kosten bei US-amerikanischen Patienten mit CAP in der stationären Pflege untersuchten. Diese Kosten lassen sich am ehesten als Behandlungspflege aus- 
Tab. 1 Merkmale der Kostenstudien zu ambulant erworbener Pneumonie (CAP).

\begin{tabular}{|c|c|c|c|c|c|}
\hline & Referenz & Land & Jahr & Studientyp & Kurzbeschreibung \\
\hline 1 & Bartolomé [3] & $\mathrm{E}$ & 2004 & prospektiv & $\begin{array}{l}\text { Studie über den Zeitraum Dezember } 1993 \text { bis November } 1995 \text { mit } \\
\text { immunkompetenten Patienten, die an CAP erkrankten. }\end{array}$ \\
\hline 2 & Bauer [4] & D & 2005 & prospektiv & $\begin{array}{l}\text { Antibiotika-Vergleichsstudie von Januar bis Juli } 2003 \text { an Patienten mit } \\
\text { CAP. }\end{array}$ \\
\hline 3 & Birnbaum [5] & USA & 2001 & retrospektiv & $\begin{array}{l}\text { Identifikation von Erwerbstätigen aus Arbeitgeberkrankenkassen- } \\
\text { daten anhand von ICD-9-Diagnosen, die im Jahre } 1997 \text { an einer Pneu- } \\
\text { monie erkrankten. }\end{array}$ \\
\hline 4 & Colice [6] & USA & 2004 & retrospektiv & $\begin{array}{l}\text { Identifikation von Erwerbstätigen aus Arbeitgeberkrankenkassen- } \\
\text { daten anhand von ICD-9-Diagnosen, die im Zeitraum von } 1996 \text { bis } \\
1998 \text { an einer CAP erkrankten. }\end{array}$ \\
\hline 5 & Estrada [7] & USA & 2000 & retrospektiv & $\begin{array}{l}\text { Durchführung einer Kohorten-Studie in einem Krankenhaus. Betrach- } \\
\text { tungszeitraum waren die Monate Januar } 1996 \text { bis März } 1997 .\end{array}$ \\
\hline 6 & Frei [8] & USA & 2004 & retrospektiv & $\begin{array}{l}\text { Analyse von Krankenakten für den Zeitraum November } 1999 \text { bis April } \\
2000 \text { in einem US-amerikanischen Krankenhaus. }\end{array}$ \\
\hline 7 & Guest [9] & UK & 1997 & retrospektiv & $\begin{array}{l}\text { Schätzung der Krankheitskosten von CAP auf Basis eines prävalenz- } \\
\text { basierten Ansatzes. Grundlage war das Datenjahr 1992/1993. }\end{array}$ \\
\hline 8 & Henderson [10] & AUS & 1997 & prospektiv & $\begin{array}{l}\text { Kostenanalyse auf Basis des Jahres } 1995 \text { für Patienten, die in die } \\
\text { Intensivpflege-Station eines Krankenhauses eingewiesen wurden. }\end{array}$ \\
\hline 9 & Kaplan [11] & USA & 2002 & retrospektiv & $\begin{array}{l}\text { Identifikation von Patienten für das Jahr } 1997 \text { aus Medicare-Versiche- } \\
\text { rungsdaten anhand von ICD-9-Diagnosen, die an CAP erkrankten. }\end{array}$ \\
\hline 10 & Kruse [12] & USA & 2003 & prospektiv & $\begin{array}{l}\text { Identifikation von Pflegebedürftigen für den Zeitraum April } 1997 \text { bis } \\
\text { September } 1998 \text { in } 36 \text { Pflegeheimen, die an CAP erkrankten, aber } \\
\text { nicht hospitalisiert wurden. }\end{array}$ \\
\hline 11 & Lave [13] & USA & 1996 & retrospektiv & $\begin{array}{l}\text { Analyse von Patienten für das Jahr 1991, die nach ICD-9-CM an CAP } \\
\text { erkrankten. Die geschätzten durchschnittlichen Kosten lagen aus } \\
\text { Sicht der Krankenhäuser deutlich unter der Honorierung. }\end{array}$ \\
\hline 12 & Niederman [14] & USA & 1998 & retrospektiv & $\begin{array}{l}\text { Analyse von Medicare Abrechnungsdaten für das Jahr } 1995 \text { und } \\
\text { Schätzung der durchschnittlichen Krankenhauskosten. }\end{array}$ \\
\hline 13 & Orrick [15] & USA & 2004 & prospektiv & $\begin{array}{l}\text { Antibiotika-Studie für den Zeitraum } 1999 \text { bis } 2000 \text { über erwachsene } \\
\text { Patienten in Florida. }\end{array}$ \\
\hline 14 & Paladino [16] & USA & 2007 & retrospektiv & $\begin{array}{l}\text { Analyse von Patienten mit hospitalisierter Pneumokokken-CAP für } \\
\text { das Jahr 2004, die auf eine ambulante Versorgung nicht ansprachen. }\end{array}$ \\
\hline 15 & Scott [17] & NZ & 2004 & retrospektiv & $\begin{array}{l}\text { Schätzung der Krankheitskosten durch CAP mithilfe eines Modells für } \\
\text { das Jahr } 2003 .\end{array}$ \\
\hline 16 & Sun [18] & USA & 2004 & retrospektiv & Kostenanalyse für den Zeitraum 1999 bis 2003 in einem Krankenhaus. \\
\hline
\end{tabular}

CAP: community acquired pneumonia

ICD-9: International Statistical Classification of Diseases and Related Health Problems, 9th Revision

ICD-9-CM: International Statistical Classification of Diseases and Related Health Problems, 9th Revision, Clinical Modification

legen. Es fanden sich keine Publikationen, die das erhöhte Risiko der Pflegebedürftigkeit nach einer CAP beleuchteten.

Die Krankheitskosten der Pneumonie lassen sich in eine Mengen-, Struktur- und Preiskomponente aufteilen. Die Menge der Inanspruchnahme von Gesundheitsleistungen hängt von der Anzahl der Krankheitsepisoden in einer Periode ab, die wiederum von der Bevölkerungsstruktur (mit den wesentlichen Einflüssen Alter und Risikofaktoren) und der Neuerkrankungsrate abhängt (siehe Abb. 2). Die Krankheitsverläufe bzw. -schwere beeinflussen die Struktur der Inanspruchnahme von Leistungen (Strukturkomponente) erheblich. Leichte Krankheitsverläufe können in vielen Fällen ambulant versorgt werden, während schwere Krankheitsverläufe regelmäßig einer stationären Behandlung bedürfen. Darüber hinaus geht ein signifikanter Einfluss von der Organisation des jeweiligen nationalen Gesundheitswesens auf die Struktur der Inanspruchnahme von Leistungen aus.

Der hier kurz erläuterte wichtige Zusammenhang zwischen Mengen- und Strukturkomponente findet in den identifizierten Kostenanalysen der CAP kaum Berücksichtigung. Die Autoren dokumentieren vorrangig Durchschnittskosten und machen keine Angaben über die Inanspruchnahme von Leistungen. Somit lassen sich Erkenntnisse aus internationalen Studien nur sehr stark eingeschränkt auf Deutschland übertragen.
Für die Publikationen zur hrQoL ist festzuhalten, dass nahezu alle identifizierten Publikationen hrQoL als primären ökonomischen Outcome in Kosten-Effektivitäts-Studien verwenden. Ziel dieser Studien war es, unterschiedliche Optionen der Antibiotika-Therapie zu vergleichen. Zwischen den einzelnen Studiengruppen ließen sich keine statistisch signifikanten Unterschiede hinsichtlich der hrQoL ermitteln. Weiterführende Analysen über die hrQoL bei CAP, wie beispielsweise in Bezug auf unterschiedliche Schweregrade, Alter und Ko-Morbidität, wurden in den identifizierten Publikationen nicht dokumentiert.

\section{Schlussfolgerung}

Die systematische Übersichtsarbeit zeigt, dass nur wenige und zudem veraltete Daten über die Krankheitskosten der CAP vorliegen und dass sich aufgrund der unterschiedlichen Studiendesigns die Ergebnisse nicht auf Deutschland übertragen lassen. Die Situation in Bezug auf die Datenlage zur hrQoL sieht noch schlechter aus. In Anbetracht der Höhe der geschätzten Kosten und der großen Anzahl Betroffener ist ein akuter Forschungsbedarf für Deutschland angezeigt. 
Tab. 2 Ergebnisse der Kostenstudien zu ambulant erworbener Pneumonie (CAP).

\begin{tabular}{|c|c|c|c|c|c|c|c|}
\hline & Referenz & Land & Jahr & Studienperspektive & Stichprobe & Kostenart & Kosten \\
\hline 1 & Bartolomé [3] & $\mathrm{E}$ & 2004 & Third-party Payer & 224 CAP & $\begin{array}{l}\text { ambulant } \\
\text { stationär }\end{array}$ & $\begin{array}{l}196 \text { Euro } \\
1.553 \text { Euro }\end{array}$ \\
\hline 2 & Bauer [4] & $\mathrm{D}$ & 2005 & $\begin{array}{l}\text { Leistungserbringer } \\
\text { (Krankenhaus) }\end{array}$ & 580 stationäre CAP & stationär & 1.333 USD \\
\hline 3 & Birnbaum [5] & USA & 2001 & $\begin{array}{l}\text { Third-party Payer } \\
\text { (Arbeitgeber) }\end{array}$ & $\begin{array}{l}1.362 \\
\text { Arbeitnehmer mit } \\
\text { CAP }(18-65 \text { Jahre })\end{array}$ & $\begin{array}{l}\text { direkt } \\
\text { ambulant \& stationär } \\
\text { indirekt }\end{array}$ & $\begin{array}{l}14.837 \text { USD } \\
13.502 \text { USD } \\
1.335 \text { USD }\end{array}$ \\
\hline 4 & Colice [6] & USA & 2004 & $\begin{array}{l}\text { Third-party Payer } \\
\text { (Arbeitgeber) }\end{array}$ & $\begin{array}{l}6.415 \text { CAP } \\
(0-64 \text { Jahre alt })\end{array}$ & $\begin{array}{l}\text { ambulant } \\
\text { stationär }\end{array}$ & $\begin{array}{l}466 \text { USD } \\
10.227 \text { USD }\end{array}$ \\
\hline 5 & Estrada [7] & USA & 2000 & $\begin{array}{l}\text { Leistungserbringer } \\
\text { (Krankenhaus) }\end{array}$ & 372 stationäre CAP & stationär & $\sim 8.000$ USD \\
\hline 6 & Frei [8] & USA & 2004 & $\begin{array}{l}\text { Leistungserbringer } \\
\text { (Krankenhaus) }\end{array}$ & 784 CAP in ICU & stationär & $\begin{array}{l}\text { 10.073 USD severe } \\
\text { 3.537 USD non-severe }\end{array}$ \\
\hline 7 & Guest [9] & UK & 1997 & Third-party Payer & 261.000 CAP-Fälle & $\begin{array}{l}\text { ambulant } \\
\text { stationär }\end{array}$ & $\begin{array}{l}100 \mathrm{GBP} \\
1.700 \mathrm{GBP}-5.100 \mathrm{GBP}\end{array}$ \\
\hline 8 & Henderson [10] & AUS & 1997 & $\begin{array}{l}\text { Leistungserbringer } \\
\text { (Krankenhaus) }\end{array}$ & 27 CAP in ICU & stationär & rd. 19.460 AUD \\
\hline 9 & Kaplan [11] & USA & 2002 & Third-party Payer & $\begin{array}{l}623.718 \text { stationäre } \\
\text { CAP }\end{array}$ & stationär & 6.949 USD \\
\hline 10 & Kruse [12] & USA & 2003 & $\begin{array}{l}\text { Leistungserbringer } \\
\text { (Pflegeheim) }\end{array}$ & $\begin{array}{l}502 \text { CAP in stationärer } \\
\text { Pflege }\end{array}$ & Pflege & $\begin{array}{l}458 \text { USD } \\
\text { Behandlungspflege }\end{array}$ \\
\hline 11 & Lave [13] & USA & 1996 & Third-party Payer & $\begin{array}{l}36.222 \text { stationäre } \\
\text { CAP }\end{array}$ & stationär & 12.467 USD \\
\hline 12 & Niederman [14] & USA & 1998 & Third-party Payer & & stationär & $\begin{array}{l}7.166 \text { USD (> } 65 \mathrm{~J} .) \\
6.042 \text { USD (<65 J.) }\end{array}$ \\
\hline 13 & Orrick [15] & USA & 2004 & $\begin{array}{l}\text { Leistungserbringer } \\
\text { (Krankenhaus) }\end{array}$ & 99 stationäre CAP & stationär & $\begin{array}{l}3.490 \text { USD } \\
\text { (ohne ärztliche Leis- } \\
\text { tungen) }\end{array}$ \\
\hline 14 & Paladino [16] & USA & 2007 & $\begin{array}{l}\text { Leistungserbringer } \\
\text { (Krankenhaus) }\end{array}$ & $\begin{array}{l}122 \text { stationäre Pneu- } \\
\text { mokokken-CAP }\end{array}$ & stationär & 12.678 USD \\
\hline 15 & Scott [17] & NZ & 2004 & Gesellschaftlich & & $\begin{array}{l}\text { direkt } \\
\text { indirekt }\end{array}$ & $\begin{array}{l}1.095 \text { NZD } \\
1.244 \text { NZD }\end{array}$ \\
\hline 16 & Sun $[18]$ & USA & 2004 & $\begin{array}{l}\text { Leistungserbringer } \\
\text { (Krankenhaus) }\end{array}$ & $\begin{array}{l}168 \text { Pneumokokken- } \\
\text { CAP }\end{array}$ & stationär & 8.654 USD \\
\hline
\end{tabular}

AUD: Australische Dollar; CAP: community acquired pneumonia; GBP: Pfund Sterling; hrQoL: health related Quality of Life ; ICU: Intensive Care Unit; J.: Jahre; NZD: Neuseeland Dollar; rd.: rund; USD: US-Dollar

Tab. 3 Merkmale der Studien zu gesundheitsbezogenen Lebensqualität bei ambulant erworbener Pneumonie (CAP).

\begin{tabular}{|c|c|c|c|c|c|c|c|c|}
\hline & Referenz & Land & Jahr & Studiengruppe & $\varnothing$ Alter & Messinstrument & Zeitraum & Kurzbeschreibung \\
\hline 1 & $\begin{array}{l}\text { Carratala } \\
{[22]}\end{array}$ & $E$ & 2005 & 224 CAP & & $\begin{array}{l}\text { PSI } \\
\text { SF-36 }\end{array}$ & 30 Tage & $\begin{array}{l}\text { Vergleich im Zeitraum Oktober } \\
2000 \text { bis Oktober } 2002 \text { von oraler } \\
\text { versus intravenöser Gabe von } \\
\text { Levofloxacin. }\end{array}$ \\
\hline 2 & $\begin{array}{l}\text { el Moussaoui } \\
{[25]}\end{array}$ & NL & 2006 & 102 CAP & 65 & $\begin{array}{l}\text { CAP-Score } \\
\text { PSI } \\
\text { SF-36 }\end{array}$ & 18 Monate & $\begin{array}{l}\text { Betrachtung von CAP-Patienten im } \\
\text { Zeitraum zwischen } 2000 \text { und } 2003 . \\
\text { Eine vollständige Erholung stellte } \\
\text { sich nach sechs Monaten ein. }\end{array}$ \\
\hline 3 & Gleason [27] & USA & 1997 & 864 CAP & & SF-36 & 30 Tage & $\begin{array}{l}\text { Analyse klinischer Endpunkte der } \\
\text { Antibiotika-Therapie. }\end{array}$ \\
\hline 4 & Marrie [21]* & CA & 2000 & 1.743 CAP & 64 & $\begin{array}{l}\text { PSI } \\
\text { SF-36 }\end{array}$ & $\begin{array}{l}6 \text { Wochen } \\
\text { post Therapie }\end{array}$ & $\begin{array}{l}\text { Vergleich von Patienten in „Critical } \\
\text { Pathway Einrichtungen“ mit kon- } \\
\text { ventionellen Gesundheitseinrich- } \\
\text { tungen. }\end{array}$ \\
\hline 5 & Torres [28] & int. & 2003 & 577 CAP & $\sim 50$ & $\begin{array}{l}\text { PSI } \\
\text { SF-36 }\end{array}$ & $\begin{array}{l}28-35 \text { Tage } \\
\text { post Therapie }\end{array}$ & $\begin{array}{l}\text { Vergleich unterschiedlicher Thera- } \\
\text { pieformen, wobei keine Differenzen } \\
\text { nachgewiesen werden konnten. }\end{array}$ \\
\hline
\end{tabular}

CAP: community acquired pneumonia; int.: international; PSI: pneumonia severity index; SF-36: Medical Outcomes Study 36-items short form; *: Feagan (2001) [19] und Palmer et al. (2000) [20] beziehen sich auf dieselbe Studiengruppe. 


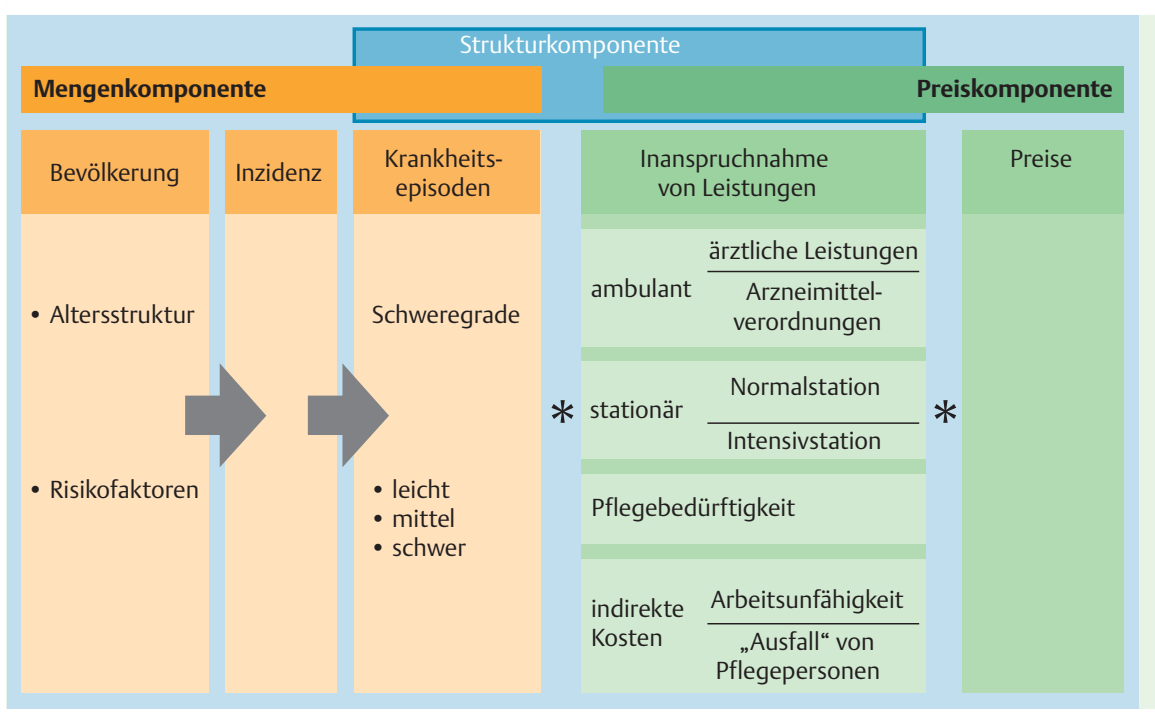

Abb. 2 Mengen-, Struktur- und Preiskomponente von Krankheitskosten.

\section{Interessenkonflikt}

$\nabla$

Der korrespondierende Autor weist auf folgende Beziehungen hin: Die Durchführung der Literaturübersicht und die Erstellung des Artikels wurden von Pfizer Deutschland mit Projektmitteln unterstützt.

\section{Literatur}

1 Höffken G, Lorenz J, Kern Wet al. Epidemiologie, Diagnostik, antimikrobielle Therapie und Management von erwachsenen Patienten mit ambulant erworbenen unteren Atemwegsinfektionen sowie ambulant erworbener Pneumonie - Update 2009. Pneumologie 2009; 63: e1 - e68

2 CAPNETZ. CAP Definition und Epidemiologie. http://www.capnetz.de/ html/practitioners/cap/definition Stand: 3. Januar 2011

3 Bartolome M, Almirall J, Morera J et al. A population-based study of the costs of care for community-acquired pneumonia. Eur Respir J 2004; 23: $610-616$

4 Bauer TT, Welte T, Ernen $C$ et al. Cost analyses of community-acquired pneumonia from the hospital perspective. Chest 2005; 128: 22382246

5 Birnbaum HG, Morley M, Greenberg PE et al. Economic burden of pneumonia in an employed population. Arch Intern Med 2001; 161: $2725-$ 2731

6 Colice GL, Morley MA, Asche C et al. Treatment costs of communityacquired pneumonia in an employed population. Chest 2004; 125 : $2140-2145$

7 Estrada CA, Unterborn JN, Price J et al. Judging the effectiveness of clinical pathways for pneumonia: the role of risk adjustment. Effective Clinical Practice 2000; 3: 221 - 228

8 Frei CR, Burgess D. Community-acquired pneumonia: Objective criteria to predict severe disease. J Infect Dis Pharmacother 2004; 6: 39- 48

9 Guest JF, Morris A. Community-acquired pneumonia: the annual cost to the National Health Service in the UK. Eur Respir J 1997; 10: 1530 1534

10 Henderson A, Cleary M, Galbraith G et al. Prospective study of costs and outcome in a major adult Australian intensive care unit utilising the APACHE III severity scoring and prediction tool. Clinical Intensive Care 1997; 8: 58-62

11 Kaplan V, Angus DC, Griffin MF et al. Hospitalized community-acquired pneumonia in the elderly: age- and sex-related patterns of care and outcome in the United States. Am J Respir Crit Care Med 2002; 165: $766-772$

12 Kruse RL, Boles KE, Mehr DR et al. The cost of treating pneumonia in the nursing home setting. J Am Med Dir Assoc 2003; 4: 81 - 89

13 Lave JR, Fine MJ, Sankey SS et al. Hospitalized pneumonia: Outcomes, treatment patterns, and costs in urban and rural areas. Journal of General Internal Medicine 1996; 11: 415-421

14 Niederman MS, McCombs JS, Unger AN et al. The cost of treating community-acquired pneumonia. Clin Ther 1998; 20: 820-837
15 Orrick JJ, Segal R, Johns TE et al. Resource use and cost of care for patients hospitalised with community acquired pneumonia: Impact of adherence to Infectious Diseases Society of America guidelines. PharmacoEconomics 2004; 22: 751 - 757

16 Paladino JA, Adelman MH, Schentag JJ et al. Direct costs in patients hospitalised with community-acquired pneumonia after non-response to outpatient treatment with macrolide antibacterials in the US. PharmacoEconomics 2007; 25: 677-683

17 Scott G, Scott H, Turley $M$ et al. Economic cost of community-acquired pneumonia in New Zealand adults. New Zealand Medical Journal 2004; 117 (1196): U933

18 Sun HK, Nicolau DP, Kuti JL. Resource utilization of adults admitted to a large urban hospital with community-acquired pneumonia caused by Streptococcus pneumoniae. Chest 2006; 130: 807-814

19 Feagan $B G$. A controlled trial of a critical pathway for treating community-acquired pneumonia: the CAPITAL study. Community-Acquired Pneumonia Intervention Trial Assessing Levofloxacin. Pharmacotherapy $2001 ; 21$ (7 Pt 2): 89S-94S

20 Palmer CS, Zhan C, Elixhauser A et al. Economic assessment of the community-acquired pneumonia intervention trial employing levofloxacin. Clinical Therapeutics 2000; 22: 250-264

21 Marrie TJ, Lau CY, Wheeler SL et al. A controlled trial of a critical pathway for treatment of community-acquired pneumonia. CAPITAL Study Investigators. Community-Acquired Pneumonia Intervention Trial Assessing Levofloxacin. JAMA 2000; 283: 749-755

22 Carratala JF-S. Outpatient care compared with hospitalization for community-acquired pneumonia: A randomized trial in low-risk patients. Annals of Internal Medicine 2005; 142: 165 - 172

23 Fine MJ, Auble TE, Yealy DM et al. A prediction rule to identify low-risk patients with community-acquired pneumonia. N Engl J Med 1997; 336: $243-250$

24 Ware JE, Kosinsky M, Keller SD. SF-36 Physical and Mental Health Summary Scales: A User's Manual. Boston: Health Assessment Laboratory, New England Medical Center; 1994

25 el Moussaoui R, Opmeer BC, de Borgie CA et al. Long-term symptom recovery and health-related quality of life in patients with mild-tomoderate-severe community-acquired pneumonia. Chest 2006; 130: $1165-1172$

26 el Moussaoui R, Opmeer BC, Bossuyt PM et al. Development and validation of a short questionnaire in community acquired pneumonia. Thorax 2004; 59: 591 - 595

27 Gleason PP, Kapoor WN, Stone RA et al. Medical outcomes and antimicrobial costs with the use of the American Thoracic Society Guidelines for outpatients with community-acquired pneumonia. Journal of the American Medical Association 1997; 278: 32 - 39

28 Torres A, Muir JF, Corris $P$ et al. Effectiveness of oral moxifloxacin in standard first-line therapy in community-acquired pneumonia. European Respiratory Journal 2003; 21: 135-143 